\title{
Lactobacillus oris
}

National Cancer Institute

\section{Source}

National Cancer Institute. Lactobacillus oris. NCI Thesaurus. Code C123493.

A species of Gram-positive, rod shaped, facultatively anaerobic bacteria in the phylum Firmicutes. This species is nonmotile and non spore forming, catalase negative, and oblig ately heterofermentative, producing both D- and L-lactic acid. L. oris is a normal flora component of the human oral cavity and gastrointestinal tract. 